\title{
Evidence for the participation of nitric oxide in pemphigus
}

\author{
M.X. Siebra ${ }^{1}$, M.A. Santos ${ }^{1}$, \\ T.L.P. Almeida ${ }^{1}$, \\ A.C.R.M. Leite ${ }^{2}$, F.Q. Cunha ${ }^{3}$ \\ and F.A.C. Rocha ${ }^{1}$
}

\author{
'Departamento de Medicina Clínica, Hospital Universitário Walter Cantídio, \\ ${ }^{2}$ Laboratório de Investigação em Osteoartropatias, Departamento de Fisiologia e \\ Farmacologia, Universidade Federal do Ceará, Fortaleza, CE, Brasil \\ 32Departamento de Farmacologia, Faculdade de Medicina de Ribeirão Preto, \\ Universidade de São Paulo, Ribeirão Preto, SP, Brasil
}

\begin{abstract}
Correspondence
F.A.C. Rocha

Departamento de Medicina Clínica

Faculdade de Medicina, UFC

Rua Dr. José Lourenço, 1930

60115-281 Fortaleza, CE

Brasil

Fax: +55-85-3244-6215

E-mail: arocha@ufc.br

Research supported by CAPES.

Publication supported by FAPESP.

$\ldots \ldots \ldots \ldots \ldots \ldots$

Received April 29, 2005

Accepted January 2, 2006

Abstract

Pemphigus is an inflammatory autoimmune disorder of the skin. Nitric oxide (NO) is an inflammatory mediator linked to a variety of physiological and pathophysiological phenomena that include skin tumors, psoriasis, urticaria, and atopic dermatitis. Inflammatory cells present in pemphigus lesions are important sources of NO production. We investigated whether $\mathrm{NO}$ is involved in pemphigus. A prospective cohort study was conducted at the Dermatology Service of the Hospital Universitário Walter Cantídio of the Federal University of Ceará. All patients seen at the outpatient clinic between August 2000 and July 2001 , with a clinically and histologically confirmed diagnosis of pemphigus were included. The median age was 42.5 years (range: $12-$ 69 years) with a male to female ratio of 3:2. Total serum nitrite levels, used as a marker for NO production, were determined by the Griess reaction. Skin biopsies from pemphigus and breast surgery (control) patients were used for the detection of the inducible NO synthase (iNOS) by immunohistochemistry. Twenty-two (22) patients with pemphigus and eight (8) controls who did not differ in demographic characteristics were included. Total serum nitrite levels were significantly higher $(>7 \mu \mathrm{mol} / \mathrm{L})$ in pemphigus patients compared to controls $(<6 \mu \mathrm{mol} / \mathrm{L})$, regardless of the severity of the clinical activity of pemphigus $(\mathrm{P}<0.0001)$. All pemphigus biopsies presented increased immunostaining for iNOS that was not detected in normal skin samples. These data are the first to demonstrate that pemphigus patients display increased serum NO levels that are associated with increased iNOS expression in the affected skin.
\end{abstract}

Pemphigus is an autoimmune disease characterized by bullous lesions involving the epidermis that affects both the skin and the oral mucosa. Clinical varieties include pemphigus vulgaris and pemphigus foliaceus. Despite the differences in their clinical presentations, these conditions appear to share a common pathogenetic mechanism that involves the participation of inflammatory
Key words - Autoimmunity

- Inflammation

- Neutrophils

- Nitric oxide

- Pemphigus

- Inducible nitric oxide synthase mediators such as the proinflammatory cytokines, tumor necrosis factor- $\alpha$ and interleukin-6 (1). Apparently, the development of autoantibodies in a particular host triggers an immunoinflammatory reaction that leads to skin damage. Antidesmoglein antibodies have been described in pemphigus patients and a direct pathogenic role for these antibodies has been proposed (2). Injection of 
anti-desmoglein antibodies into the skin of animals led to the development of lesions similar to the clinical picture seen in patients with pemphigus (3). Typical lesions consist of epidermal blisters filled with a prominent cell influx that includes lymphocytes, macrophages, neutrophils, and eosinophils that surround the acantholytic cell (4).

Nitric oxide (NO) is produced by $\mathrm{NO}$ synthase (NOS) enzymes, using L-arginine as substrate. Endothelial (NOS3) and neuronal (NOS1) NOS isoforms are constitutive, whereas NOS2 is the inducible isoform (iNOS). The production of small amounts of NO via constitutive NOS activation in neuronal cells, platelets and endothelial cells has been linked to homeostasis, whereas sustained and large amounts of NO produced after iNOS activation in inflammatory cells such as neutrophils and macrophages, after being triggered by inflammatory stimuli, have been linked to pathophysiological phenomena (5). The detection of increased NO levels has been regarded as evidence for the participation of NO in many autoimmune diseases, including rheumatoid arthritis and systemic lupus erythematosus (6). NO also appears to be involved in a variety of skin diseases such as skin cancers (7), psoriasis (8), urticaria (9), and atopic dermatitis (10).

To our knowledge, there are no reports in the literature showing the participation of NO in pemphigus. Since inflammatory cells are important sources of $\mathrm{NO}$ and they also mediate tissue damage in pemphigus, it seemed reasonable to propose that NO participates in the pathogenesis of this disease. In the present study, we provide histochemical evidence that pemphigus patients present increased expression of iNOS in the lesioned skin that is associated with increased serum nitrite levels.

A prospective cohort study was conducted at the Hospital Universitário Walter Cantídio of the Faculty of Medicine of the Federal University of Ceará. An outpatient clinical service was established in order to provide assistance to all patients presenting bullous dermatosis. All patients seen between $\mathrm{Au}$ gust/2000 and July/2001 were prospectively entered in the present study. The Hospital Ethics Committee approved the protocol and all patients signed an informed consent form.

Patients with a diagnosis of pemphigus based on clinical and routine histological studies were included in the study. The patients were seen by two experienced dermatologists for clinical examination (M.A.S. and T.L.P.). All patients were subjected to fasting blood sampling in order to perform routine exams that included complete blood cell counts, erythrocyte sedimentation rate, serum creatinine, prothrombin activity, and $\mathrm{C}$ reactive protein. A type I urine exam was also performed. Serum samples were kept at $-70^{\circ} \mathrm{C}$ for the determination of total nitrite, as a measure of NO production, using the Griess reaction (see below).

All patients were subjected to a skin biopsy as part of the routine diagnostic evaluation. Briefly, skin specimens were collected using a punch biopsy device. After embedding in paraffin, 6- $\mu \mathrm{m}$ sections of this material were processed for hematoxylin-eosin staining and examined by light microscopy.

Healthy controls of the same average age as the patients were used for the determination of serum nitrite levels. Healthy skin tissue from breast specimens, obtained from patients undergoing plastic surgery, was used as a control for the histopathological studies.

Determination of $\mathrm{NO}$ production. Production of $\mathrm{NO}$ was determined by measuring plasma total nitrite plus nitrate $\left(\mathrm{NO}_{2}{ }^{-}\right.$ plus $\mathrm{NO}_{3}^{-}$) by the Griess reaction. Total $\mathrm{NO}_{2}^{-}$plus $\mathrm{NO}_{3}{ }^{-}$levels were determined after the $\mathrm{NO}_{3}{ }^{-}$in $0.08-\mathrm{mL}$ samples was converted to $\mathrm{NO}_{2}^{-}$by incubation of $0.01 \mathrm{~mL}$ nitrate reductase from Aspergillus species (1 unit $/ \mathrm{mL}$ ) and $0.01 \mathrm{~mL} / \mathrm{mM}$ NADPH for $30 \mathrm{~min}$ at $37^{\circ} \mathrm{C}$. $\mathrm{NO}_{2}{ }^{-}$levels were determined spectrophotometrically at $540 \mathrm{~nm}$ by measuring the absorbance of test samples 
(0.1 mL) after adding $0.1 \mathrm{~mL}$ Griess reagent (sulfanilic acid (1\% w/v) and N-(1-naphythyl)ethylenediamine $(0.1 \mathrm{w} / \mathrm{v})$ in $5 \%$ phosphoric acid) and comparing these values with those from standard solutions of $\mathrm{NaNO}_{2}$ (1-100 $\mu \mathrm{M})$. Data are reported as nitrate plus nitrite concentration and were analyzed by ANOVA, followed by the Tukey test, with the level of significance set at $\mathrm{P}<0.05$.

Immunohistochemistry for the detection of $i N O S$. The skin samples from the pemphigus patients and controls were used to assess the expression of the iNOS enzyme, using a commercially available rabbit anti-human iNOS monoclonal antibody (Santa Cruz Biotechnology, Santa Cruz, CA, USA). Briefly, after deparaffinization, the tissues were incubated with hydrogen peroxide and washed. After incubation in $0.01 \mathrm{M}$ citrate buffer, $\mathrm{pH}$ 6.0 , the tissues were heated in a microwave oven. After overnight unspecific blocking with rabbit serum, the samples were incubated for $2 \mathrm{~h}$ with the rabbit anti-iNOS antibody diluted 1:100 in PBS plus 1\% BSA. After rinsing, the sections were incubated with a secondary biotinylated anti-rabbit IgG antibody. The reaction product was detected with the avidin-biotin-peroxidase complex (Vector Laboratories, Burlingame, CA, USA) and the color of the reaction product was detected by reaction with diamino-benzidine tetrahydrochloride (Sigma, St. Louis, MO, USA). The sections were counterstained with Mayer hematoxylin.

Demographic and clinical data of patients and control subjects. Twenty-two patients were considered eligible to enter the study. The median age was 42.5 years (range: $12-69$ years). Eight $(36.4 \%)$ of the patients were females and 14 (63.6\%) were males, with a male to female ratio of $3: 2$. Seven patients had inactive disease on the basis of no demonstrable active cutaneous or mucosal lesions. Twenty patients (90.9\%) were receiving oral steroids (mean \pm SD dose of $30.7 \pm$ $31.2 \mathrm{mg} /$ day). There were no patients on immunosuppressive drugs other than steroids.
Serum levels of $\mathrm{NO}_{2}^{-}$plus $\mathrm{NO}_{3}^{-}$of patients with pemphigus. Figure 1 shows that $18(81.8 \%)$ of the patients with pemphigus had total nitrite levels higher than $7 \mu \mathrm{mol} / \mathrm{L}$, as opposed to less than $6 \mu \mathrm{mol} / \mathrm{L}$ in healthy individuals. The values obtained for normal subjects were similar to those reported in the literature (11) and the differences between groups were significant $(\mathrm{P}<0.0001)$. There were no significant differences in serum nitrite plus nitrate levels between pemphigus vulgaris and foliaceus patients. Furthermore, there was no statistically significant difference in serum nitrite plus nitrate levels between patients considered to have clinically active or inactive pemphigus.

Detection of iNOS expression by immunohistochemistry. Figure 2A and B illustrates skin biopsies stained with hematoxylin-eosin of a healthy control (A) and a pemphigus patient (B), respectively. Figure 2C shows the immunohistochemistry of a normal skin biopsy showing no specific iNOS staining. Figure 2D is representative of a skin biopsy sample from a pemphigus patient showing intense and diffuse iNOS immunostaining. The immunostaining is particularly evident in the epidermal layers compared to the appearance of the normal skin (Figure 2A and C). All skin biopsy samples obtained from pemphigus patients tested (10 samples) exhibited similar results, though exhibiting variability in the intensity of iNOS expression (data not shown).

Figure 1. Serum NO levels in pemphigus patients. Fasting blood samples were collected from patients and controls. After centrifugation, serum was used for the determination of total nitrate plus nitrite levels using the Griess reaction. Data are reported as means \pm SEM of serum nitrate plus nitrite levels $(\mu \mathrm{M})$ of each subject. Control, healthy subjects $(\mathrm{N}=8)$; PV-A, active pemphigus vulgaris $(\mathrm{N}=$ 7); PF-A, active pemphigus foliaceus $(\mathrm{N}=8)$; $P V-I$, inactive pemphigus vulgaris $(\mathrm{N}=3)$; PF-I, inactive pemphigus foliaceus $(\mathrm{N}=4)$. ${ }^{*} \mathrm{P}<0.05$ compared to control (ANOVA followed by Tukey test). 
The results presented here demonstrate for the first time that pemphigus patients display increased serum levels of NO, assessed as the total nitrate + nitrite levels. The increased expression of the iNOS isoenzyme in skin specimens of pemphigus patients suggests that activation of this NOS isoform is responsible for this phenomenon.

Pemphigus is an autoimmune disease of rare occurrence. In populations of the north-

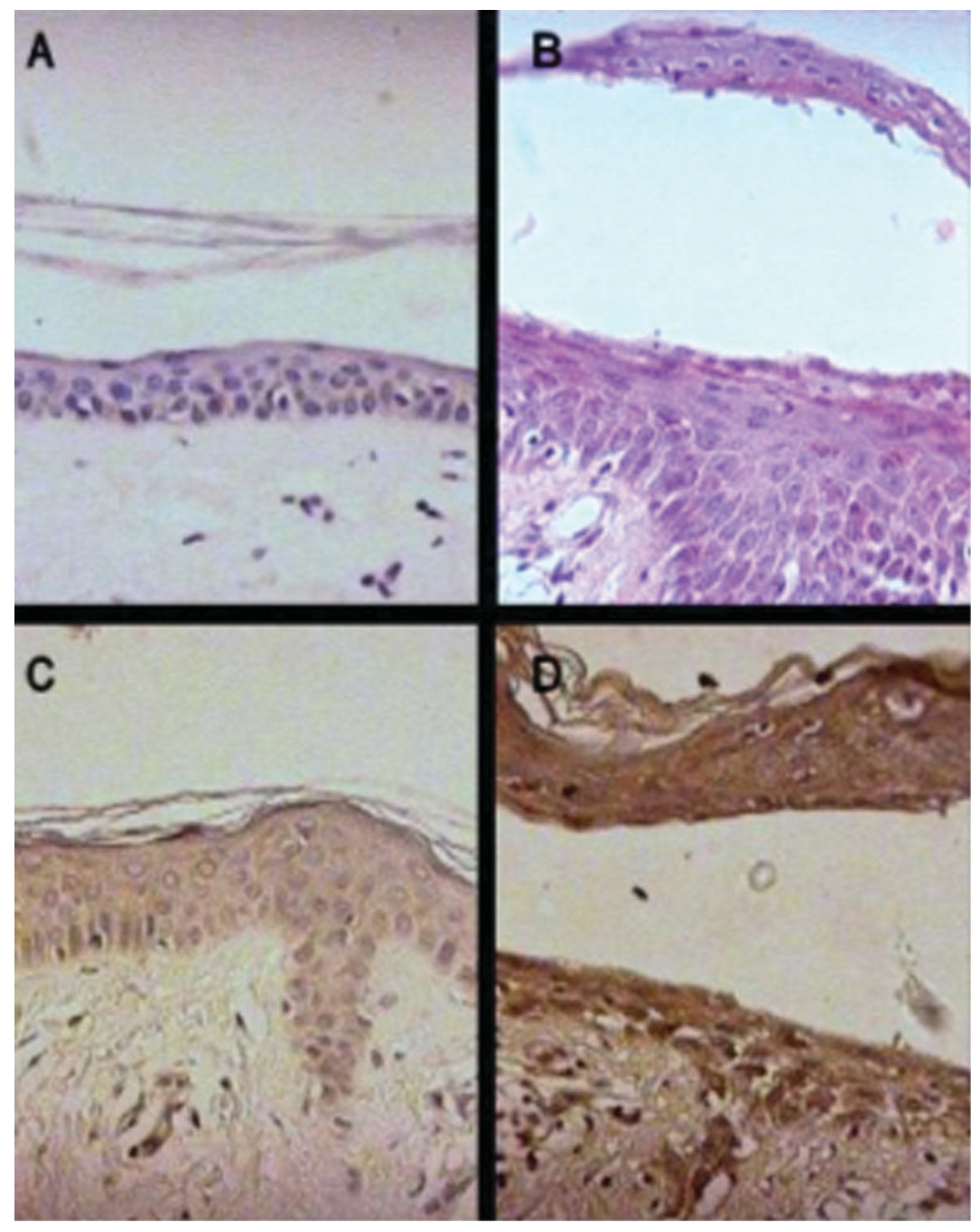

Figure 2. Representative light micrographs of immunohistochemistry for the detection of iNOS in a skin biopsy from healthy controls and pemphigus patients. All pemphigus skin specimens displayed similar staining. Figure $2 A$ and $B$ illustrates skin biopsies stained with hematoxylin-eosin of a healthy control (A) and a pemphigus patient (B). Figure $2 \mathrm{C}$ and $\mathrm{D}$ illustrates the immunohistochemistry of skin samples obtained from a healthy person (C) and a pemphigus patient (D) showing intense and diffuse iNOS immunostaining in the latter, that was particularly evident in the epidermal layers, as compared to the appearance of the healthy skin (original magnification 400X). ern hemisphere, the prevalence ranges from 0.4 to $1.6 / 100,000$ inhabitants (12). There are no data on the prevalence of this disease in Brazil. The occurrence of pemphigus, popularly called "fogo selvagem" (wildfire), is considered endemic in the western part of the country (13). The present study was conducted in Fortaleza, State of Ceará, which is located in the northeast of Brazil $\left(4^{\circ} \mathrm{S} / 38^{\circ}\right.$ E). Fortaleza has 2,219,836 inhabitants (Brazilian Institute of Geography and Statistics IBGE, 2002) (14) and most of them are considered to be descendants of white Portuguese and native Brazilians, i.e., descendants from the Indians that lived in Brazil before the European colonization. Fortaleza is located about $2,000 \mathrm{~km}$ northeast of the regions of endemic occurrence of the "fogo selvagem". The Service of Dermatology of the Hospital Universitário Walter Cantídio, Faculty of Medicine of the Federal University of Ceará, is a dermatological reference center for all patients seeking the public health system (about $75 \%$ of the population). All patients included in the present study are residents of Fortaleza. Hence, considering the population at risk and the number of patients included, our series is probably representative of our region.

The demonstration of the participation of NO in pemphigus points to a role for this substance in mediating tissue damage in this disease. Interestingly, both resident skin cells and the infiltrating inflammatory cells, including neutrophils, strongly stained for iNOS. Also, there was no significant difference between patients considered to have active or inactive disease. This observation may indicate that the clinical parameters currently used to monitor pemphigus do not necessarily reflect silent disease activity. In fact, it is not unusual to see pemphigus patients considered to have inactive disease showing exacerbation, regardless therapy.

The regulation of iNOS expression is multifactorial. Despite the use of steroids by most of our patients, increased iNOS expres- 
sion was detected in all patient samples compared to normal skin samples. Unfortunately, the demonstration of iNOS expression by immunohistochemistry is not quantitative and thus the effect of steroids on enzyme expression cannot be measured.

NO has been shown to participate in a diverse array of diseases. Apparently, the deleterious effects of NO might be linked, at least in part, to the reactive nitrogen species generated following NO release (15). Under inflammatory conditions, large amounts of $\mathrm{NO}$ and of the superoxide $\left(\mathrm{O}_{2}^{-}\right)$anion are produced, leading to the formation of a strong oxidant, the peroxynitrite anion. The proposed cytotoxic properties of peroxynitrite include protein nitration, lipid peroxidation, inhibition of cellular metabolic pathways and signal transduction mechanisms, and
DNA strand breaks (16). Formation of 3nitrotyrosine from the reaction of peroxynitrite with proteins has been used as a biological marker for peroxynitrite formation in vivo (17). 3-Nitrotyrosine detected in atherosclerotic plaques (18), in the serum and synovial fluid of rheumatoid arthritis patients (19), and in rejected human kidney allografts (20) is one of the examples given as evidence of the association of peroxynitrite formation with human disease states.

On the basis of the present demonstration of the association of NO with pemphigus, we propose that NO may be indirectly involved in mediating tissue damage in this disease, probably via the production of reactive nitrogen species. We are currently testing this hypothesis.

\section{References}

1. Amagai M (1995). Adhesion molecules. I: Keratinocyte-keratinocyte interactions; cadherin and pemphigus. Journal of Investigative Dermatology, 104: 146-152.

2. Amagai M, Karpati S, Prussick R et al. (1992). Autoantibodies against the amino-terminal cadherin-like domain of pemphigus vulgaris are pathogenic. Journal of Clinical Investigation, 90: 919-926.

3. Anhalt GJ, Labib RS, Voorhees JJ et al. (1982). Induction of pemphigus in neonatal mice by passive transfer of IgG from patients with disease. New England Journal of Medicine, 306: 1189-1196.

4. Hietanen J (1982). Clinical and cytological features of oral pemphigus. Acta Odontologica Scandinavica, 40: 403-414.

5. Moncada S \& Palmer RMJ (1990). The L-arginine:nitric oxide pathway in the vessel wall. In: Moncada S \& Higgs EA (Editors), Nitric Oxide from L-Arginine: A Bioregulatory System. Elsevier, Amsterdam, The Netherlands, 19-33.

6. Abramson SB, Amin AR, Clancy RM et al. (2001). The role of nitric oxide in tissue destruction. Best Practice and Research. Clinical Rheumatology, 15: 831-845.

7. Kagoura M, Matsui C, Toyoda M et al. (2001). Immunohistochemical study of inducible nitric oxide syntase in skin cancers. Journal of Cutaneous Pathology, 28: 476-481.

8. Sirsjo A, Karlsson M, Gidlof A et al. (1996). Increased expression of inducible nitric oxide synthase in psoriatic skin and cytokine-stimulated cultured keratinocytes. British Journal of Dermatology, 134: 643-648.

9. Becherel PA, Chosidow O, Le Goff L et al. (1997). Inducible nitric oxide synthase and proinflammatory cytokine expression by human keratinocytes during acute urticaria. Molecular Medicine, 3: 686-694.

10. Taniuchi S, Kojima T, Hara Mt K et al. (2001). Increased serum nitrate levels in infants with atopic dermatitis. Allergy, 56: 693-695.
11. Moshage H, Kok B, Huizenga JR et al. (1995). Nitrite and nitrate determinations in plasma: a critical evaluation. Clinical Chemistry, 41: 892-896.

12. Stanley JR (1999). Pemphigus. In: Fitzpatrick TB, Eisen AZ \& Wolff K (Editors), Dermatology in General Medicine. 5th edn. Vol. 1. McGraw-Hill, Philadelphia, PA, USA, 654-666.

13. Diaz LA, Sampaio SAP, Rivitti EA et al. (1989). Endemic pemphigus foliaceus (fogo selvagem). II. Current and historic epidemiologic studies. Journal of Investigative Dermatology, 92: 4-12.

14. Instituto Brasileiro de Geografia e Estatística - Perfil dos Municípios Brasileiros - 2002.

15. Pryor WA \& Squadrito GL (1995). The chemistry of peroxynitrite: a product from the reaction of nitric oxide and superoxide. American Journal of Physiology, 268: L699-L722.

16. Beckman JS, Chen J, Ischiropoulos $\mathrm{H}$ et al. (1994). Oxidative chemistry of peroxynitrite. Methods in Enzymology, 233: 229-240.

17. Van Der Vliet A, Eiserich JP, Kaur H et al. (1996). Nitrotyrosine as a biomark for reactive nitrogen species. Methods in Enzymology, 269: 175-184.

18. Beckman JS, Ye YZ, Anderson PG et al. (1994). Extensive nitration of protein tyrosines in human atherosclerosis detected by immunohistochemistry. Biological Chemistry, 375: 81-88.

19. Kaur H \& Halliwell B (1994). Evidence for nitric oxide-mediated oxidative damage in chronic inflammation. Nitrotyrosine in serum and synovial fluid from rheumatoid patients. FEBS Letters, 350: 912.

20. MacMillan-Crow LA, Crow JP, Kerby JD et al. (1996). Nitration and inactivation of manganese superoxide dismutase in chronic rejection of human renal allografts. Proceedings of the National Academy of Sciences, USA, 93: 11853-11858. 